\title{
Recombinant Adenovirus Vector Bearing Antisense Macrophage Migration Inhibitory Factor cDNA Prevents Acute Lipopolysaccharide-Induced Liver Failure in Mice
}

\author{
Takayuki Iwaki, Motoi Sugimura, Jun Nishihira, Toshiki Matsuura, Takao Kobayashi, \\ and Naohiro Kanayama \\ Department of Obstetrics and Gynecology (TI, MS, TM, TK, NK), Hamamatsu University School of Medicine, \\ Hamamatsu, and Central Research Institute (JN), Hokkaido University School of Medicine, Sapporo, Japan
}

\begin{abstract}
SUMMARY: Macrophage migration inhibitory factor (MIF) is a pleiotropic cytokine involved in delayed hypersensitivity and cellular immunity. MIF also acts as a proinflammatory cytokine and counterregulates the anti-inflammatory effects of glucocorticoids. Exogenous gene transfer mediated by adenovirus is useful to study a particular molecular function as well as to develop gene therapy strategies. A recombinant adenovirus containing sense and antisense murine MIF (mMIF) cDNA inserts was constructed using a cosmid-terminal protein complex method. The sense mMIF adenovirus (AxCA-mMIFS) efficiently induced MMIF in COS-7 cells that endogenously lack MMIF in a dose-dependent manner. In contrast, the antisense mMIF adenovirus (AxCA-mMIFAS) inhibited the expression of mMIF in NIH3T3 cells in a dose-dependent manner. To assess the pathophysiologic role of MIF in acute liver failure, we induced acute onset of liver damage in mice (male Jcl:ICR) by a combined treatment of Bacille Calmette-Guerin (BCG) and lipopolysaccharide (LPS). mMIF level in the liver of mice infected with AxCA-mMIFAS showed a significant reduction in MIF production in response to BCG-LPS compared with mice treated without viral infection and with AxCA-mMIFS. In addition, the immunohistochemical staining demonstrated that F4/80 antigen on macrophage was enhanced in liver infected with AxCA-mMIFS but reduced in liver infected with AxCA-mMIFAS. The staining intensity is correlated with the mMIF antigen level in liver tissue. The survival rate of mice infected with AxCA-mMIFAS was significantly higher than that of mice treated with PBS and infected with AxCA-LacZ in BCG-LPS. These results suggest that inhibition of MIF production, using recombinant adenovirus bearing the antisense MIF gene, reduced the mortality rate in BCG-LPS-induced liver failure in mice. This finding might aid in the further development of gene therapy targeting MIF. (Lab Invest 2003, 83:561-570).
\end{abstract}

A cute liver failure is an uncommon condition associated with a high mortality rate; it requires liver transplantation in more than $50 \%$ of cases (Ostapowicz and Lee, 2000). The histopathologic studies in the liver reveal a prominent infiltration of lymphocytes with massive necrosis. Enhanced inflammatory reaction in response to various stimuli such as viral infection or drug toxicity has been implicated in the pathogenesis of liver failure (Bernal and Wendon, 1999). In the pathology of hepatic damage and regeneration, various cytokines play an important role (Simpson, 1999). Administration of a small dose of lipopolysaccharides (LPS) in mice pretreated with Bacille Calmette-Guerin (BCG) induces acute and massive hepatic necrosis (Shands and Senterfitt, 1981). This acute liver failure model is associated with a $70 \%$ mortality within several hours of administrating LPS. Histologically, a

DOI: 10.1097/01.LAB.0000062857.26210.EF

Received December 5, 2002.

This study was supported by a grant from Japan Society for the Promotion of Science.

Address reprint requests to: Dr. Motoi Sugimura, Handayama 1-20-1, Hamamatsu, Japan.E-mail:msugi@hama-med.ac.jp marked infiltration of monocytes around necrotic lesions that is similar to those observed in acute liver failure in humans is evident (Ferluga, 1981; Ferluga and Allison, 1978; Mizoguchi et al, 1988). Recent studies using this model have demonstrated that cytokines such as TNF- $\alpha$ (Tokushige et al, 2000; Tsutsui et al, 1997), IL-1 $\beta$ (Fujioka et al, 1995; Liu et al, 1990), and interferon- $\gamma$ (Okamura et al, 1996) may be involved in the pathogenesis of the development of acute liver failure.

Macrophage migration inhibitory factor (MIF) was the first lymphokine to be discovered (Bloom and Bennett, 1966). Elevated expression of MIF is correlated with delayed hypersensitivity and cellular immunity in humans (David, 1966). MIF genes of various species have been isolated and characterized (Mitchell et al, 1995; Paralkar and Wistow, 1994; Sakai et al, 1994; Weiser et al, 1989). Recently, the molecular functions of MIF were reported (Bucala, 1996; Nishihira, 2000). MIF is a pleiotropic cytokine released from the anterior pituitary gland during systemic inflammation and seems to play a central role in the toxic response to endotoxemia and possibly septic shock (Bernhagen et al, 1993; Calandra et al, 1995). In 
endotoxemia, MIF acts as a proinflammatory cytokine and counterregulates the anti-inflammatory effects of glucocorticoids (Leech et al, 2000). At present, it has been demonstrated that various cells or tissues, other than that of the immune system, express MIF (Abe et al, 2000; Arcuri et al, 1999; Nishihira et al, 1998; Wada et al, 1997).

BCG up-regulates the expression of MIF mRNA in the liver. This increase can be enhanced by treatment with LPS after BCG priming (Kobayashi et al, 1999). It is of note that the mortality rate after treatment with anti-MIF antibody is significantly diminished (Bernhagen et al, 1993). However, the role of MIF as a proinflammatory agent is still under debate (Honma et al, 2000; Kleemann et al, 2000). In this study, we constructed recombinant adenoviruses containing sense and antisense MIF cDNAs to determine whether the level of MIF influences the development of BCGprimed LPS-induced acute liver failure in mice.

\section{Results}

\section{Construction of Recombinant Adenoviruses}

The orientation of MIF cDNA inserted in the Swal site of pAxCAwt was determined by PCR, and two cosmids were obtained (Fig. 1). The recombinant adenoviruses AxCA-mMIFS and AxCA-mMIFAS contained forward and reverse inserts (Miyake et al, 1996), respectively (Fig. 2). MIF insert was amplified by PCR with the primer set 1 (mMIF-5' and mMIF-3') as described in "Materials and Methods." The length of the PCR products was $440 \mathrm{bp}$. Both adenoviruses were positive for the primer set 1 . The insert was amplified with either the primer set 2 (AxCAwt-5' and mMIF-3') or set 3 (AxCAwt-5' and mMIF-5'). The length of these PCR products was 512 bp. AxCAmMIFS and pAxCA-mMIFS were positive with the primer set 2 and negative with the primer set 3 . However, AxCA-mMIFAS and pAxCA-mMIFAS showed the opposite result (Fig. 3). DNA sequences of PCR products were bidirectionally analyzed by a DNA sequence analyzer ( $\mathrm{ABI} 310$ ) (data not shown). The two recombinant adenovirus constructs were di- gested with Xhol and analyzed by electrophoresis. No extra bands derived from parent and/or mutated viruses were observed (data not shown).

\section{Effect of AxCA-MIFS and AxCA-MIFAS on MIF Protein Levels}

Our preliminary data showed that COS-7 cells did not produce mMIF, whereas NIH3T3 cells produced relatively high amounts of mMIF. Thus, we transfected COS-7 cells with AxCA-mMIFS and NIH3T3 cells with AxCA-mMIFS or AxCA-mMIFAS. MIF protein level in COS-7 cells transfected with AxCA-mMIFS increased in a dose-dependent manner (Fig. 4). The protein level of MIF in NIH3T3 cells transfected with AxCA-mMIFAS decreased in a dose-dependent manner; accordingly, cells transfected with AxCA-mMIFS showed that the level of MIF increased (Fig. 5). There were no differences in the total cell counts or in the total cellular proteins in both groups (data not shown).

\section{Effect of the AxCA-mMIFS and the AxCA-mMIFAS on Survival Rate in Mice Challenged with BCG and LPS}

Mice transfected with AxCA-mMIFS and primed with BCG 7 days before LPS injection had a significantly lower survival rate 48 hours after LPS injection (3\%) compared with the survival rate of mice transfected with AxCA-mMIFAS (92\%; Fig. 6). The mice transfected with AxCA-mMIFAS were more resistant to LPS challenge than mice treated with PBS or with AxCALacZ. Survival rate in mice treated with PBS and transfected with AxCA-LacZ was better than that in mice transfected only with AxCA-mMIFS.

\section{MIF Protein Levels in the Liver}

mMIF protein levels in the liver of mice treated with BCG/LPS/AxCA-mMIFS was significantly elevated relative to mice treated with BCG alone (Fig. 7). In contrast, mMIF protein level in mice treated with BCG/AxCA-mMIFAS or with BCG/LPS/AxCAmMIFAS were significantly reduced compared with that with BCG alone or with BCG/LPS. However, there

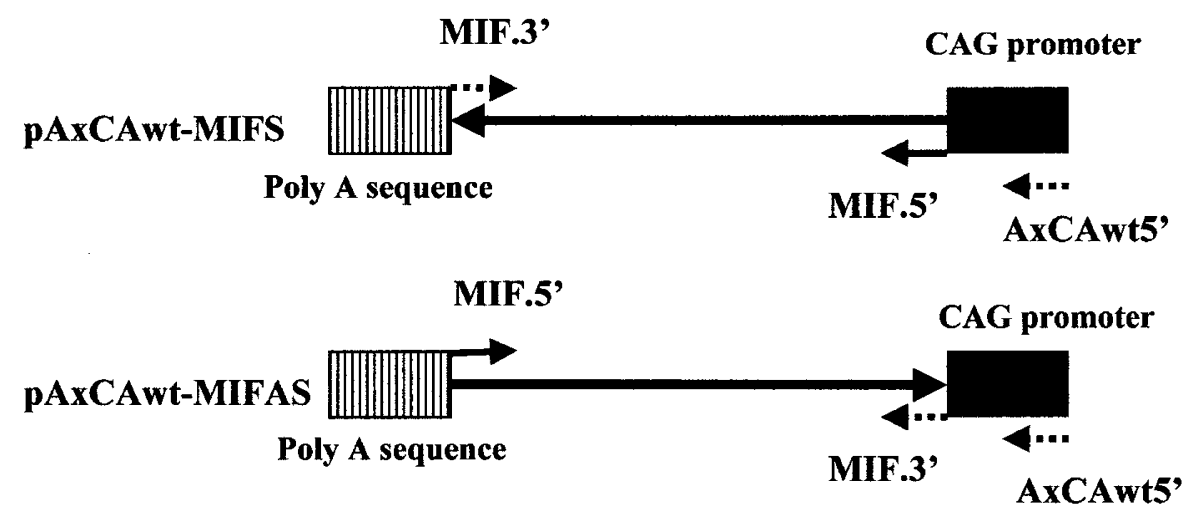

Figure 1.

Insertion of murine MIF cDNA into the cassette cosmid. The orientation of MIF CDNA insert in the cosmid was determined by PCR and sequence analysis, and the two cosmid vectors pAxCAwt-MIFS and pAxCAwt-MIFAS were obtained containing forward and reverse inserts of MIF cDNA, respectively. 


\section{mMIF cDNA with CAG promoter and polyA sequence}

\section{AxCA-MIFS}

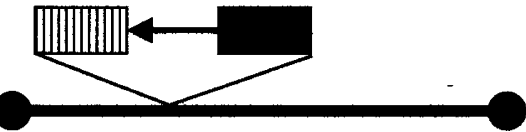

mMIF cDNA with CAG promoter and polyA sequence

\section{AxCA-MiFAS}

\section{Figure 2.}

Construction of recombinant adenoviruses. Recombinant adenoviruses were generated by in vivo homologous recombination in 293 cells between the cosmids and EcoT22I-digested Ad5-dIX DNA tagged with viral terminal proteins. The arrows show the orientation of the transcription. A triangle under the adenovirus genome represents a deletion of an adenovirus sequence. Transfection was carried out using the calcium phosphate method.

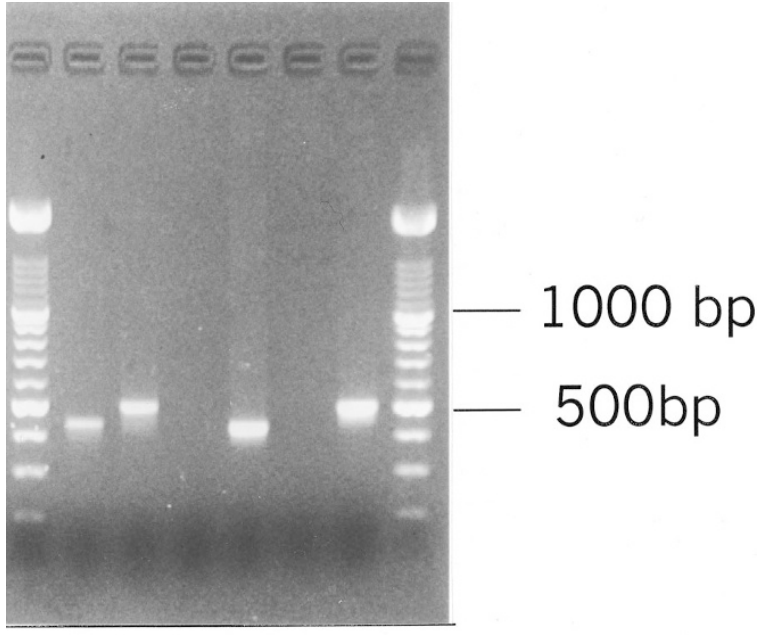

M $123123 \mathrm{M}$

AxCA-MIFS AxCA-MIFAS

Figure 3.

Analysis of insert and orientation of recombinant adenoviruses. The length of these PCR products was $512 \mathrm{bp}$. AxCA-mMIFS and pAxCA-mMIFS showed positive bands with the primer set 2 but not with the primer set 3. However, AxCA-mMIFAS and pAxCA-mMIFAS showed positive bands with the primer set 3 but not with the primer set 2. $M$, molecular marker.

was no significant difference in MMIF level between $\mathrm{BCG} / \mathrm{LPS}$ in the presence or absence of AxCA-mMIFS.

\section{Histologic Examination and Morphometric Analyses}

Histologic changes in the liver of mice treated with BCG alone, with BCG/recombinant adenoviruses, with BCG/LPS, or with BCG/LPS/recombinant adenoviruses were assessed. Liver tissues from mice treated with BCG/LPS (Fig. 8B) showed degenerated hepatocytes with vacuoles and marked infiltration of mononuclear cells at necrotic lesion sites compared with those treated with BCG alone (Fig. 8A). Quantitative analysis of the necrotic area was $19.0 \pm 2.3 \%(n=3)$ in the liver treated with BCG/LPS; however, no ne-

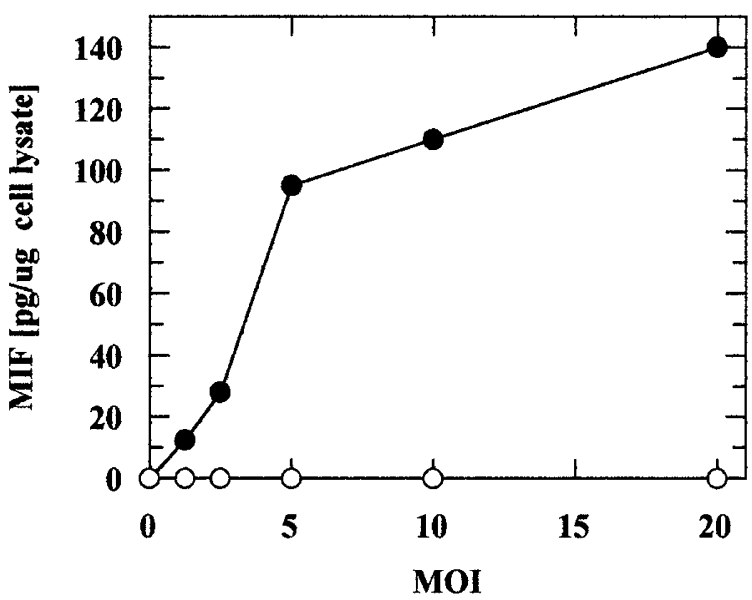

Figure 4.

MMIF level in COS-7 cells transfected with AxCA-mMIFS. MIF protein content in COS-7 cells was determined by ELISA for MIF after transfection with AxCA-mMIFS (๑) and AxCA-LacZ (O). Each point represents the mean value of two independent experiments.

crotic area was detected in the liver treated with BCG alone. The necrotic changes in response to BCG/LPS (Fig. 8B) were enhanced in mice transfected with AxCA-mMIFS (Fig. 8E). This necrotic area was $32.4 \pm$ $2.8 \%$ and significantly higher than without AxCAmMIFS. In contrast, the histologic changes induced by BCG/LPS (Fig. 8B) were minimal in liver tissue from mice transfected with AxCA-mMIFAS (Fig. 8F). This necrotic area was $0.8 \pm 0.8 \%(n=3)$ and significantly smaller than those treated with BCG and LPS. No significant necrotic changes were observed in liver tissues treated with BCG alone (Fig. 8A), with BCG/ AxCA-mMIFS (Fig. 8C), or with BCG/AxCA-mMIFAS (Fig. 8D).

\section{Immunohistochemistry}

Immunohistochemical staining of $F 4 / 80$ antigen in livers of mice treated with BCG or BCG and LPS in the presence or absence of recombinant adenovirus was examined. Positive staining of F4/80 antigen was detected in infiltrated mononuclear cells in the livers of 


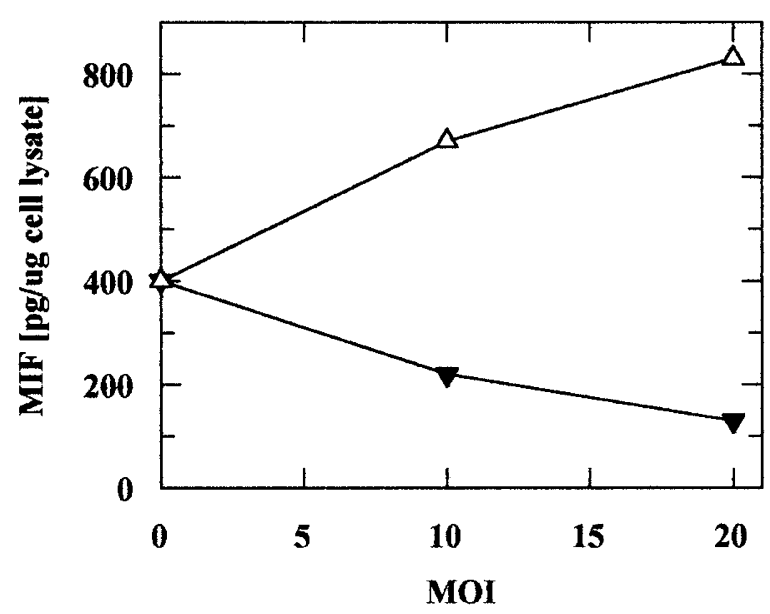

Figure 5.

MMIF protein level in NIH3T3 cells transfected with AxCA-mMIFS and AXCA-mMIFAS. mMIF protein contents in NIH3T3 cells transfected with AxCA-mMIFS ( $\mathbf{\Delta}$ ) and AxCA-mMIFAS $(\boldsymbol{v})$ were determined by ELISA. The numbers of NIH3T3 cells transfected with AxCA-mMIFS $(\boldsymbol{\Delta})$ and AxCAmMIFAS ( $\mathbf{v})$ were equivalent at confluent culture conditions. Each point represents the mean value of two independent experiments.

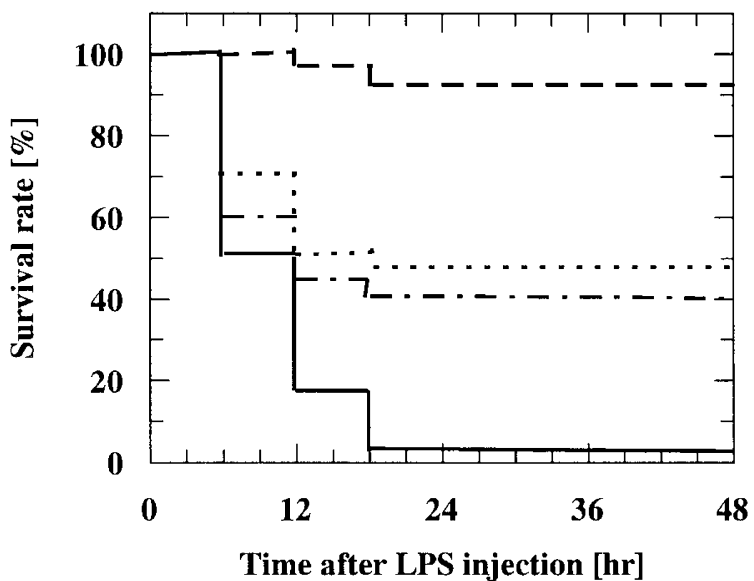

Figure 6.

Effect of AxCA-mMIFS and AxCA-mMIFAS on the survival rate in mice primed with BCG and challenged with LPS. Each group of 30 mice received an injection of $2.0 \times 10^{9}$ pfu of AxCA-mMIFS, AxCA-mMIFAS, AxCA-LacZ, or PBS $\left(\mathrm{Ca}^{2+}[-], \mathrm{Mg}^{2+}[-]\right)-10 \%$ glycerol buffer in addition to injection of BCG before LPS challenge to examine the effect of AxCA-mMIFS and AxCA-mMIFAS on BCG-LPS-induced acute liver failure. The survival rate at 48 hours after 10 $\mu \mathrm{g}$ of LPS injection after 7 days of priming with BCG was significantly high in mice infected with AxCA-mMIFAS (- - ) compared with the survival rate in mice infected with AxCA-mMIFS (-). The mice infected with AxCA-mMIFAS were more resistant to LPS challenge than mice treated with PBS (----) and infected with AxCA-LacZ $(-\cdot \cdot \cdot-)$. The mice treated with AxCA-mMIFS were more lethal to LPS challenge than mice with PBS and AxCA-LacZ. $p<0.001$ for difference between AxCA-mMIFAS $(n=30)$ and AxCA-LacZ $(n=30)$, AxCA-mMIFAS $(n=30)$ and AxCA-mMIFS $(n=30) . p<0.01$ for difference between AxCA-mMIFS $(n=30)$ and AxCA-LacZ $(n=30)$.

mice in all treatment (Fig. 9). The staining intensity in liver treated with BCG/AxCA-mMIFS (Fig. 9C) was enhanced compared with BCG/AxCA-mMIFAS (Fig. 9D). Staining at necrotic lesions was diffuse and distinct in tissues treated with BCG/LPS (Fig. 9B) and with BCG/LPS/AxCA-mMIFS (Fig. 9E). In contrast, the staining of $F 4 / 80$ antigen was weak in mice treated with BCG/LPS/AxCA-mMIFAS (Fig. 9F).

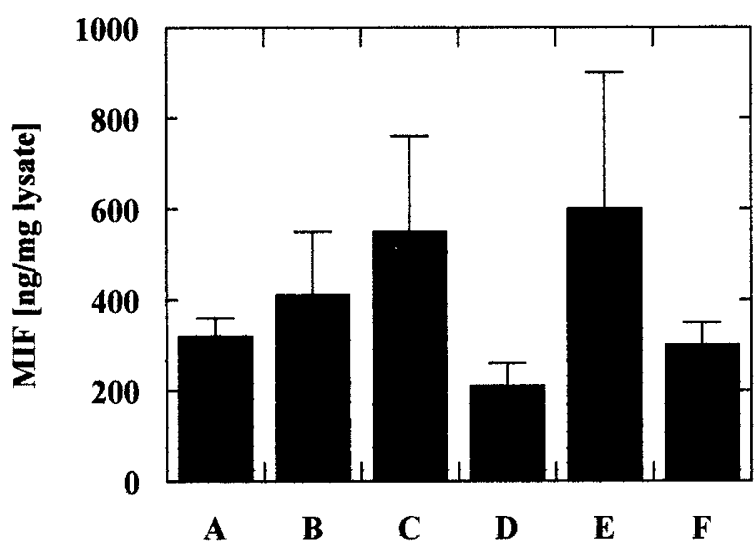

Figure 7

Effect of AxCA-mMIFS or AxCA-mMIFAS on mMIF levels in the liver tissue from mice treated with $B C G$ and LPS. A, BCG alone; B, BCG/LPS; C, BCG/AxCA-mMIFS; D, BCG/AxCA-mMIFAS; E, BCG/LPS/AxCA-MIFS; F, BCG/ LPS/AxCA-MIFAS. Each point represents the mean value \pm SE $(n=7)$. $p<$ 0.01 for differences between $\mathrm{A}$ and $\mathrm{C}, \mathrm{A}$ and $\mathrm{D}, \mathrm{B}$ and $\mathrm{F}$.

\section{Discussion}

Several reports have suggested the involvement of various cytokines, including TNF- $\alpha, \mathrm{IL}-1 \beta$, and interferon- $\gamma$, in the pathogenesis of acute liver failure (Fujioka et al, 1995; Liu et al, 1990; Okamura et al, 1996; Tokushige et al, 2000; Tsutsui et al, 1997). Within the inflammatory cytokine network, MIF has been implicated as a key molecule functioning as an initiator of glucocorticoids (Bucala, 1996). However, its precise mechanism of action remains to be elucidated. In the present study, we have shown that treatment with recombinant adenovirus, containing antisense MMIF cDNA, is effective in reducing the mortality rate in BCG-LPS-induced acute liver failure in mice. This is the first report demonstrating the role of MIF in severe inflammatory reaction using antisense MIF technology. In addition, the present data revealed that the level of preformed $\mathrm{mMIF}$ in the liver, produced by parenchymal and nonparenchymal cells, may be critical for exacerbating liver damage.

Histologic changes in the animal study showed that BCG-primed LPS stimulation enhanced the infiltration of T lymphocytes into the liver and might play a critical role in the pathogenesis of acute liver failure. We previously reported the use of anti-MIF antibody to prevent liver tissue damage in the same model (Kobayashi et al, 1999). The data presented here further support the finding that liver tissue damage could be prevented by reducing MIF production in the liver.

Among various vector systems that have been studied, the adenovirus vector proved to be clinically useful because of the simplicity of its preparation and the high efficiency of gene transduction irrespective of tissue type and cell cycle. Our preliminary study showed a high level of $\beta$-galactosidase activity in the liver tissue taken from mice transfected with AxCALacZ compared with the tissue from other organs (data not shown). This observation was compatible with a previous report showing that more than $90 \%$ of recombinant adenoviruses injected in the tail vein 

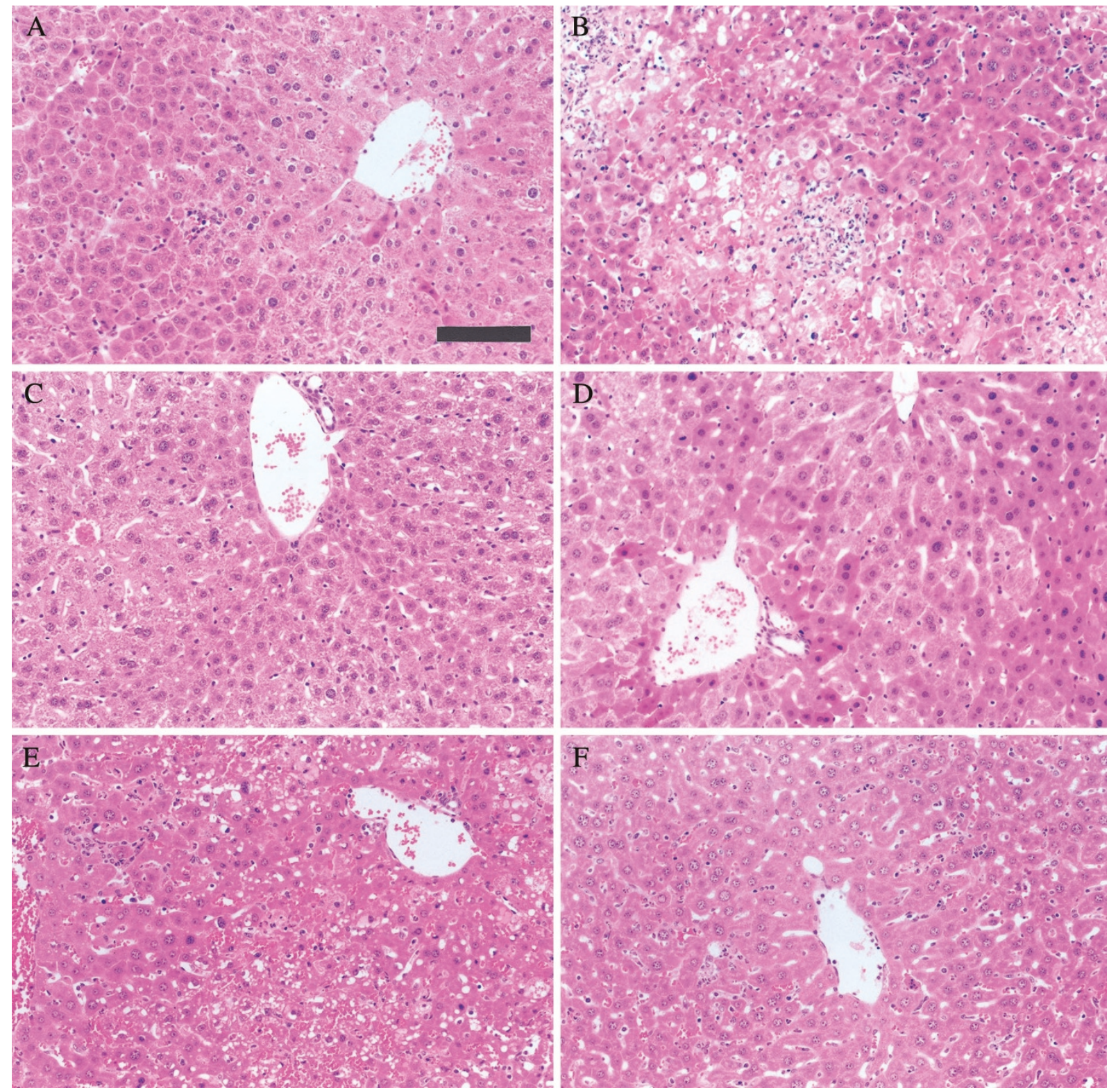

\section{Figure 8.}

Effect of AxCA-mMIFS or AxCA-mMIFAS on histologic changes in the liver of mice treated with BCG and LPS. A, BCG alone; B, BCG/LPS; C, BCG/AxCA-mMIFS; D, BCG/AxCA-mMIFAS; E, BCG/LPS/AxCA-MIFS; F, BCG/LPS/AxCA-MIFAS. The arrow indicates the marked infiltration of mononuclear cells. The scale bar in A indicates $400 \mu \mathrm{m}$ (original magnification, $\times 50$ )

were concentrated in the liver (Peeters et al, 1996). We revealed that the recombinant adenoviruses containing sense mMIF cDNA efficiently induced mMIF in COS-7 cells, which lack endogenous mMIF. We also determined that recombinant adenoviruses containing antisense mMIF cDNA (AxCA-mMIFAS) suppressed the expression of mMIF protein in NIH3T3 cells, which normally produce high levels of mMIF. mMIF protein level in the liver of mice transfected with AxCAMMIFAS showed a significant reduction of MMIF in response to BCG-primed LPS challenge compared with BCG-primed LPS treatment. The immunohistochemical staining showed that the F4/80 antigens positive macrophages were enhanced in the liver transfected with AxCA-mMIFS, whereas a reduced level of MIF was observed in mice transfected with AxCA-mMIFAS. Staining intensity was strongly correlated with the mMIF protein level in the liver. These data suggest that up-regulation of mMIF leads to enhancement of inflammatory reactions in liver damage.

In an acute liver injury, administration of a small dose of LPS into BCG-primed mice often causes a lethal hepatic injury mimicking fulminant hepatitis in humans. This injury is characterized by a marked infiltration of mononuclear cells such as Kupffer cells, macrophages, and, to a lesser extent, T cells (Matsui et al, 1997; Tanaka et al, 1996; Tsuji et al, 1997). Administration of anti-MIF antibody into mice primed with BCG serves to protect them from acute hepatic 

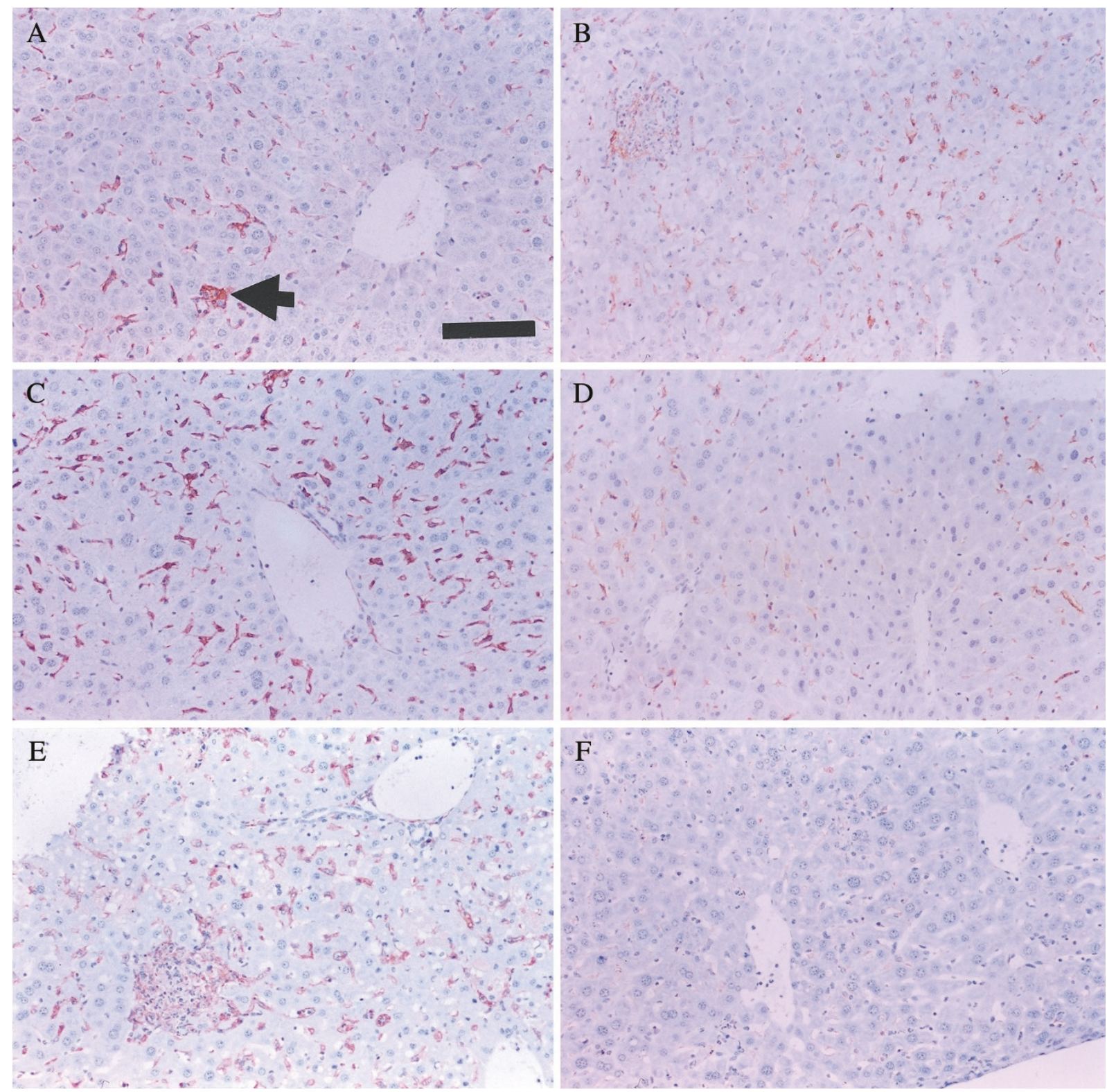

Figure 9.

Immunohistochemical localization of F4/80 antigen on infiltrated macrophages in the livers treated with BCG-primed LPS challenge in the presence or absence of transfection with AxCA-mMIFS or AxCA-mMIFAS. A, BCG alone; B, BCG/LPS; C, BCG/AxCA-mMIFS; D, BCG/AxCA-mMIFAS; E, BCG/LPS/AxCA-MIFS; F, BCG/LPS/AxCA-MIFAS. The arrow indicates the positive staining of antigen F4/80. The scale bar in A indicates $400 \mu \mathrm{m}$ (original magnification, $\times 50$ ).

failure perhaps by suppressing TNF- $\alpha$ and by preventing the infiltration of T cells (Kobayashi et al, 1999).

In this study, we found minimal histologic changes in liver treated with BCG and AxCA-MIFS, suggesting that up-regulation of MIF alone in the liver might not be sufficient to induce liver damage. The expression of MIF mRNA in the liver was increased by treatment with BCG or the combination of BCG and LPS. Marked liver tissue damage was apparent after treatment with LPS, suggesting that LPS initiates the release of preformed MIF within the cells and triggers the induction of secondary proinflammatory mediator, such as TNF- $\alpha$. This suggests that MIF is not a proinflammatory cytokine that directly affects tissue damage but may activate downstream events associated with inflammatory cascades, leading to tissue injury.

Consistent with this finding is a study demonstrating that anti-MIF antibody reduced MIF/LPS-induced mortality rate in mice (Bernhagen et al, 1993). Anti-MIF antibody prevented endotoxin-induced liver failure (Kobayashi et al, 1999) perhaps by neutralizing the bioactivity of MIF or by inhibiting the secondary induction of TNF- $\alpha$ and IL-1 in monocytes and T cells in response to MIF. This model demonstrated that histologic changes were more prominent in liver tissue than in other organs. This finding suggests that the local pathologic process in the liver may contribute to the enhanced mortality. Cooperativity of MIF and 
TNF- $\alpha$ may not always be necessary for exerting proinflammatory action of MIF. Calandra et al (2000) showed that anti-MIF antibody protected TNF- $\alpha$ knockout mice from lethal peritonitis induced by cecal ligation and puncture, suggesting a role of MIF independent of TNF- $\alpha$ in the pathogenesis of sepsis. Reports from others have suggested that coupled action between MIF and TNF- $\alpha$ is critical for severe inflammatory reactions. It was reported that mice lacking MIF gene were more resistant to LPS-induced endotoxin shock (Bozza et al, 1999). These mice were resistant to the lethal effect of high-dose LPS and had lower plasma levels of TNF- $\alpha$ than wild-type mice but have normal levels of IL-6 and IL-10. Furthermore, the role of MIF in the inflammatory reaction, such as response to endotoxin or exotoxin stimuli, has been well demonstrated. Despite the number of reports supporting the inflammatory role of MIF, conflicting results have emerged. MIF-deficient mice responded normally to LPS stimuli and had no phenotype (Honma et al, 2000). The basis for these discrepancies is not clear. The genetic background of mice and their immunologic redundancy may contribute to these differences. Additional studies, using conditional gene-disrupted mice, are necessary to elucidate the role of MIF.

In addition, that MIF functions as a proinflammatory cytokine has also been argued by a recent report (Kleemann et al, 2000), which showed that MIF may function in an anti-inflammatory capacity through binding with Jab1. MIF could suppress the biologic function of Jab1, which is a co-activator of AP-1, leading to inflammatory states. It has been hypothesized that there might be two signaling pathways for $\mathrm{MIF}$, one is specific receptor-mediated and the other is by endocytosis (Bucala, 2000). MIF at a lower dose may function as a proinflammatory cytokine through the receptor-mediated pathway, whereas it works as an anti-inflammatory molecule at higher doses through endocytosis. To the best of our knowledge, it is more likely that MIF may function to enhance inflammatory reactions in vivo according to the available data to date, including our current data using recombinant adenoviruses.

Finally, we revealed the pivotal role of MIF in acute liver failure by transfection of recombinant adenovirus. This finding suggested the possibility that a MIFtargeting strategy may be a good candidate for therapy of acute hepatic damage. Namely, using antisense MIF therapy to block the expression of MIF, ultimately inhibiting the downstream transcription pathways that are essential for inflammatory reaction. Moreover, the recombinant adenoviruses containing antisense MIF gene may contribute to identifying its precise role within the cytokine network and to elucidate the pathogenesis of acute liver failure.

\section{Materials and Methods}

\section{Insertion of Murine MIF cDNA into the Cassette Cosmid}

The murine full-length wild-type MIF cDNA (456 bp) was excised from the pBluescript II KS (-) (Stratagene
Cloning System, La Jolla, California) containing murine wild-type MIF with EcoRI and HindIII (New England Biolabs, Beverly, Massachusetts). The 456-bp EcoRIHindIII fragments were blunted with T4 DNA polymerase (Takara, Osaka, Japan) and inserted into the Swal site of pAxCAwt (Takara). The insert of mMIF cDNA and its orientation in the cosmid vector were determined by PCR and sequence analysis. Briefly, $50 \mathrm{ng}$ of cosmid was amplified in TRIS buffer $(10 \mathrm{~mm}$ Tris- $\mathrm{HCl}$, $\mathrm{pH}$ 8.3) containing $50 \mathrm{~mm} \mathrm{KCl}, 1.5 \mathrm{~mm} \mathrm{MgCl} 2,1 \mathrm{U}$ of TaqDNA polymerase (Perkin Elmer, Norwalk, Connecticut), $0.1 \mathrm{~mm}$ dNTP, and $100 \mathrm{~nm}$ of each specific primer pair in a total volume of $25 \mu$ l. The PCR was carried out for 20 cycles of denaturation at $94^{\circ} \mathrm{C}$ for 30 seconds, annealing at $60^{\circ} \mathrm{C}$ for 30 seconds, and extension at $72^{\circ} \mathrm{C}$ for 30 seconds using a thermal cycler (Model 9600; Perkin Elmer). mMIF.5' (5'GGGTCACGTAGCTCAGGTCC, position 9 to 28), mMIF.3' (5'-GCCAGGACTCAAGCGAAGGTG, position 428 to 448) and AxCAwt.5' (5'-CAACGTGCTGGTTGTTGTGCTG, position 1679 to 1700) were used as primers. Primer set 1 (mMIF.5' and mMIF.3') was used for determination of the insert, and the expected size of PCR amplicon was $440 \mathrm{bp}$. Primer set 2 (AxCAwt.5' and mMIF.3') and primer set 3 (AxCAwt.5' and mMIF.5') were used for the determination of the orientation of inserts, and the expected size of the PCR amplicon was 512 bp. The mMIF.5' was a sense primer, and the mMIF.3' was an antisense primer for mMIF. This PCR amplicon corresponded to nucleotides 9 to 448 of the mMIF cDNA sequence registered in the EMBL data bank (U20156). The AxCAwt.5' was located at the $5^{\prime}$ stream of pAxCAwt from the cloning site (Miyake et al, 1996).

\section{Preparation of Recombinant Adenoviruses}

Recombinant adenoviruses were generated by homologous recombination in HEK293 cells (Japan Health Science Foundation, Osaka, Japan) using the constructed cosmids and EcoT22I-digested Ad5-dIX DNA tagged with viral terminal proteins (Takara) (Kiwaki et al, 1996). Briefly, $8 \mu \mathrm{g}$ of the cosmids and $5 \mu \mathrm{l}$ of the EcoT22I-digested Ad5-dIX DNA were co-transfected into confluent HEK293 cells in a 6-cm tissue culture dish. Transfection was carried out using a calcium phosphate method. Twenty-four hours later, the transfected HEK293 cells were removed from the 6-cm tissue culture dish using $0.5 \mathrm{~mm}$ EDTA/PBS and resuspended in $11 \mathrm{ml}$ of DMEM/5\% FCS (culture A). Nontransfected HEK293 cells were removed from the $10-\mathrm{cm}$ tissue culture dish and resuspended in $30 \mathrm{ml}$ of DMEM/5\% FCS (culture B). One milliliter of culture A and $10 \mathrm{ml}$ of culture B were mixed, and then $100 \mu \mathrm{l}$ of the mixture was subcultured into each well of a 96-well collagen-coated tissue culture plate. The adenoviruses in the completely denatured cells from each well were extracted by cycling through a freeze/ thaw process six times, and the cell debris was removed by centrifugation (the first seed of adenoviruses). An aliquot of $10 \mu \mathrm{l}$ of the first seed of adenoviruses and $0.5 \mathrm{ml}$ of DMEM/5\% FCS was added to 
HEK293 cells in a 24-well collagen-coated tissue culture plate. After the cells were denatured, they were collected by centrifugation and the genomic and viral DNA in the cells was treated with $400 \mu$ l of lysis solution $(0.02 \mathrm{mg} / \mathrm{ml}$ Proteinase K, 0.01\% SDS, 50 mm Tris-HCl [pH 7.5], 100 $\mathrm{mm} \mathrm{NaCl}$, and $10 \mathrm{~mm}$ EDTA) for 1 hour at $50^{\circ} \mathrm{C}$. They were then extracted with two steps of phenol/chloroform extraction and eluted with $50 \mu$ l of elution buffer $(10 \mathrm{~mm}$ Tris- $\mathrm{HCl}$ [pH 8.0], $1 \mathrm{~mm}$ EDTA, and $20 \mu \mathrm{g} / \mathrm{ml}$ RNase A). An aliquot of $1 \mu \mathrm{l}$ of these DNA solutions was used for PCR with the same condition described in "Insertion of Murine MIF cDNA into the Cassette Cosmid" and $15 \mu \mathrm{l}$ then digested with Xhol (New England Biolabs), and gel electrophoresis was performed to check for contamination of unexpected viruses. After several amplification steps, the recombinant adenoviruses were purified and concentrated using two cycles of $\mathrm{CsCl}$ step gradients followed by dialysis against PBS in the absence of $\mathrm{Ca}^{2+}$ and $\mathrm{Mg}^{2+}$ containing $10 \%$ glycerol buffer as described (Kanegae et al, 1994). AxCA-LacZ was a gift from Dr. Izumu Saito and amplified and purified as described above.

\section{Titration of Recombinant Adenoviruses}

A total of $50 \mu$ l of DMEM supplemented with $5 \%$ FCS was added to each well of a 96-well collagen-coated tissue culture plate, and eight rows of 3-fold serial diluted viruses starting from $10^{-5}$ dilution were prepared. To each well, a total of $3 \times 10^{5} \mathrm{HEK} 293$ cells in $50 \mu \mathrm{l}$ of DMEM containing $5 \%$ FCS were added. The plate was incubated at $37^{\circ} \mathrm{C}$ in $5 \% \mathrm{CO}_{2}$, and $50 \mu \mathrm{l}$ of DMEM containing 10\% FCS was added to each well at days 5 and 9. Day 12 was chosen as the end point of the cytotoxic effect. The plate was microscopically examined, and the $50 \%$ tissue culture infectious dose was determined as previously described (Kanegae et al, 1994).

\section{Effect of Recombinant Adenoviruses on Culture Cells}

To assess the effect of recombinant adenoviruses, we transfected two cell types, COS-7 (Japan Health Science Foundation, Osaka, Japan) and NIH3T3 (American Type Culture Collection, Manassas, Virginia). COS-7 cells (JCRB 9127) were established from monkey kidney by transformation with an origin-defective mutant of SV40, which codes for wild-type T antigen. NIH3T3 cells (CRL-1658) were derived from murine embryo. Both cells were maintained in DMEM containing $10 \% \mathrm{FCS}, 5 \% \mathrm{CO}_{2}$ at $37^{\circ} \mathrm{C}$. Effect of AxCAmMIFS on the induction of mMIF was examined in COS-7 cells that endogenously do not express mMIF. The effect of AxCA-mMIFAS and AxCA-mMIFS on the expression of mMIF was also examined in NIH-3T3 cells that express relatively high amounts of mMIF. Briefly, $5 \times 10^{5}$ COS-7 cells were cultured in $1 \mathrm{ml}$ of DMEM containing 10\% FCS in a six-well plate for 24 hours at $37^{\circ} \mathrm{C}$ in $5 \% \mathrm{CO}_{2}$. Cells were transfected with AxCA-mMIFS from 1 to 20 multiplicity of infection and cultured for 72 hours. Similarly, NIH 3T3 cells $\left(5 \times 10^{5}\right.$ cells) were transfected with 1 to 200 multiplicity of infection of AxCA-mMIFAS and cultured for 72 hours under the same condition. After incubation, cells were harvested and lysed with lysis buffer $(50 \mathrm{~mm}$ Tris- $\mathrm{HCl}$, $\mathrm{pH}$ 7.6) containing $150 \mathrm{~mm} \mathrm{NaCl}, 10 \mathrm{~mm}$ EGTA, and $0.5 \%$ NP-40.

\section{Induction of Acute Liver Failure by BCG-Primed LPS Challenge in Mice Transfected with AxCA-mMIFS or AxCA-mMIFAS}

Male Jcl:ICR mice (5 weeks old) were purchased from Japan SLC (Hamamatsu, Japan). They were maintained under pathogen-free conditions and allowed access to food and water ad libitum. Animal research described in this study was carried out in compliance with the Declaration of Helsinki and was conducted with approval by the Animal Experiment Ethics Committee of Hamamatsu University School of Medicine. Mice received an injection of $1 \mathrm{mg} / \mathrm{body}$ BCG (Nippon BCG, Tokyo, Japan) and $2.0 \times 10^{9}$ pfu recombinant adenovirus containing sense or antisense mMIF cDNA in tail veins. After 7 days, mice received an injection of $10 \mu \mathrm{g} / \mathrm{body}$ LPS (Escherichia coli serotype 0111:B21; Sigma) in tail veins. The dose of LPS was determined by preliminary experiments in male Jcl:ICR mice that demonstrated a $50 \%$ to $60 \%$ lethal dose. Survival rate was evaluated for 48 hours after LPS challenge. In a separate series of experiments, mice were killed at various time points, and blood and tissue samples were collected and stored at $-80^{\circ} \mathrm{C}$ until use.

\section{Preparation of Lysates from Culture Cells and Tissues}

The liver tissues obtained 5 hours after LPS injection in PBS containing proteinase inhibitors (50 mM EDTA, 1 $\mathrm{mm}$ benzamidine, and $1 \mathrm{mg} / \mathrm{ml}$ leupeptin) were homogenized with a Polytron (Kinematica, Lezern, Switzerland) and centrifuged at $12000 \times g$. The transfected cells were harvested 72 hours after transfection and resuspended in the same buffer used as above. The supernatants were removed, and the total amount of protein in these supernatants was measured by BCA methods.

\section{ELISA of mMIF for Lysates from Culture Cells and Tissues}

mMIF protein levels in cell lysates and tissue specimens were measured by ELISA as described previously (Mizue et al, 2000). Briefly, $50 \mu \mathrm{l}$ of PBS containing anti-rat MIF IgG polyclonal antibody $(4 \mathrm{mg} / \mathrm{ml})$ was added to each well in a 96-well microtiter plate and incubated for 30 minutes at room temperature. After washing with distilled water, nonspecific binding was blocked with $0.5 \%$ BSA in PBS for 20 minutes at room temperature. A total of $100 \mu$ l of cell or tissue lysates from tissues was added to each well and incubated for 1 hour at room temperature. The plate was washed three times with PBS containing $0.05 \%$ Tween-20 (washing buffer), and $50 \mu \mathrm{l}$ of biotinconjugated rabbit anti-MIF antibody was added to each well. After incubation for 1 hour at room temperature, the plate was washed three times with the 
washing buffer, and the avidin-conjugated goat antirabbit IgG antibody was added to each well and incubated for 15 minutes at room temperature. After washing three times with washing buffer, the bound complex was detected by chromogenic substrate. Ten microliters of the substrate solution contained $8 \mathrm{mg}$ of o-phenylenediamine and $4 \mu \mathrm{l}$ of $30 \% \mathrm{H}_{2} \mathrm{O}_{2}$ in citrate phosphate buffer ( $\mathrm{pH}$ 5.0). After incubation for 20 minutes at room temperature, the reaction was stopped with $25 \mu \mathrm{l}$ of $4 \mathrm{~N}$ sulfuric acid. The absorbance was measured at $492 \mathrm{~nm}$ by a spectrophotometer (Model 3500; Bio-Rad, Hercules, California).

\section{Histologic Examination}

Liver tissues were fixed in $10 \%$ neutral formalin, embedded in paraffin, and sectioned at 4- $\mu \mathrm{m}$ thick. After deparaffinizing, samples were stained with hematoxylin and eosin (H\&E).

\section{Morphometric Analysis}

Morphometric measurements of liver section stained with $\mathrm{H} \& \mathrm{E}$ were performed using a computer-assisted image analysis system (Bioquant True color Windows Software; Biometrics, Nashville, Tennessee). Three different areas in $\times 50$ magnification were randomly selected, and the necrotic field in each area was measured and compared with total area. The average values per liver were determined.

\section{Immunohistochemistry}

Immunohistochemical analysis was performed using streptavidin-biotin method according to the manufacturer's protocol (Nichirei, Tokyo, Japan). Briefly, after deparaffinizing, 4- $\mu \mathrm{m}$-thick tissue sections were treated with methanol containing $0.3 \%$ hydrogen peroxide for 30 minutes to inactivate endogenous peroxidase. After washing with PBS, sections were incubated with PBS containing 10\% rat serum albumin for 30 minutes. An anti-mouse mAb against F4/80 antigen (Serotec, Kidlington, United Kingdom) was used to label macrophages (Austyn and Gordon, 1981). Tissue sections were then incubated with rat anti-mouse F4/80 antibody (1:100 dilution). After washing with PBS, sections were incubated with biotin-conjugated anti-rat IgG antibody (1:1000 dilution; Chemicon, Temecula, California). After an additional wash with PBS, sections were visualized with 3, 3'-diaminobenzidine tetrachloride containing $0.01 \%$ hydrogen peroxide for 2 minutes and counterstained with hematoxylin.

\section{Statistical Analysis}

Data were presented as a mean \pm SE unless otherwise specified. The survival rates were analyzed using a log-rank test. MIF protein levels in the lysates from cultured cells and tissues were analyzed using MannWhitney $U$ test. All analysis was performed using the computer-assisted Statview program (Abacus Concepts, Berkeley, California), and $p<0.05$ was considered to be significant.

\section{Acknowledgement}

We are grateful to Y. Mizue of Sapporo Immunodiagnostic Laboratory for assistance in the measurement of mMIF by ELISA. We also thank Dr. Angelina J. Lay for her careful reading of this article.

\section{References}

Abe R, Shimizu T, Ohkawara A, and Nishihira J (2000). Enhancement of macrophage migration inhibitory factor (MIF) expression in injured epidermis and cultured fibroblasts. Biochem Biophys Acta 1500:1-9.

Arcuri F, del Vecchio MT, de Santi MM, Lalinga AV, Pallini V, Bini L, Bartolommei S, Parigi S, and Cintorino M (1999). Macrophage migration inhibitory factor in the human prostate: Identification and immunocytochemical localization. Prostate 39:159-165.

Austyn JM and Gordon S (1981). F4/80, a monoclonal antibody directed specifically against the mouse macrophage. Eur J Immunol 11:805-815.

Bernal W and Wendon J (1999). Acute liver failure: Clinical features and management. Eur J Gastroenterol Hepatol 9:977-984

Bernhagen J, Calandra T, Mitchell RA, Martin SB, Tracey KJ, Voelter W, Manogue KR, Cerami A, and Bucala R (1993). MIF is a pituitary-derived cytokine that potentiates lethal endotoxaemia. Nature 365:756-759.

Bloom BR and Bennett B (1966). Mechanism of a reaction in vitro associated with delayed-type hypersensitivity. Science 153:80-82.

Bozza M, Satoskar AR, Lin G, Lu B, Humbles AA, Gerard C, and David JR (1999). Targeted disruption of migration inhibitory factor gene reveals its critical role in sepsis. J Exp Med 189:341-346.

Bucala R (1996). MIF re-discovered: Pituitary hormone and glucocorticoid-induced regulator of cytokine production. FASEB J 7:19-24.

Bucala R (2000). Signal transduction. A most interesting factor. Nature 408:146-147.

Calandra T, Bernhagen J, Metz CN, Spiegel LA, Bacher M, Donnelly T, Cerami A, and Bucala R (1995). MIF as a glucocorticoid-induced modulator of cytokine production. Nature 377:68-71.

Calandra T, Echtenacher B, Roy DL, Pugin J, Metz CN, Hultner L, Heumann D, Mannel D, Bucala R, and Glauser MP (2000). Protection from septic shock by neutralization of macrophage migration inhibitory factor. Nat Med 6:164-170.

David JR (1966). Delayed hypersensitivity in vitro: Its mediation by cell-free substances formed by lymphoid cell-antigen interaction. Proc Natl Acad Sci USA 56:72-77.

Ferluga J (1981). Tuberculin hypersensitivity hepatitis in mice infected with Mycobacterium bovis (BCG). Am J Pathol 105:82-90.

Ferluga $\mathrm{J}$ and Allison AC (1978). Role of mononuclear infiltrating cells in pathogenesis of hepatitis. Lancet 2:610-611.

Fujioka N, Mukaida N, Harada A, Akiyama M, Kasahara T, Kuno K, Ooi A, Mai M, and Matsushima K (1995). Preparation of specific antibodies against murine IL-1ra and the establishment of IL-1ra as an endogenous regulator of bacteriainduced fulminant hepatitis in mice. J Leukoc Biol 58:90-98. 
Honma N, Koseki H, Akasaka T, Nakayama T, Taniguchi M, Serizawa I, Akahori H, Osawa M, and Mikayama T (2000). Deficiency of the macrophage migration inhibitory factor gene has no significant effect on endotoxaemia. Immunology 100 : 84-90.

Kanegae Y, Makimura M, and Saito I (1994). A simple and efficient method for purification of infectious recombinant adenovirus. Jpn J Med Sci Biol 47:157-166.

Kiwaki K, Kanegae Y, Saito I, Komaki S, Nakamura K, Miyazaki Jl, Endo F, and Matsuda I (1996). Correction of ornithine transcarbamylase deficiency in adult spf (ash) mice and in OTC-deficient human hepatocytes with recombinant adenoviruses bearing the CAG promoter. Hum Gene Ther 7:821-830.

Kleemann R, Hausser A, Geiger, G. Mischke R, BurgerKentischer A, Flieger O, Johannes FJ, Roger T, Calandra T, Kapurniotu A, Grell M, Finkelmeier D, Brunner H, and Bernhagen $J$ (2000). Intracellular action of the cytokine MIF to modulate AP-1 activity and the cell cycle through Jab1. Nature 408:211-216.

Kobayashi S, Nishihira J, Watanabe S, and Todo S (1999). Prevention of lethal acute hepatic failure by antimacrophage migration inhibitory factor antibody in mice treated with Bacille Calmette-Guerin and lipopolysaccharide. Hepatology 29:1752-1759.

Leech M, Metz C, Bucala R, and Morand EF (2000). Regulation of macrophage migration inhibitory factor by endogenous glucocorticoids in rat adjuvant-induced arthritis. Arthritis Rheum 43:827-833.

Liu P, Ohnishi H, Moriwaki H, and Muto Y (1990). Enhanced tumor necrosis factor and interleukin-1 in an experimental model of massive liver cell necrosis/fatal hepatitis in mice. Gastroenterol Jpn 25:339-342.

Matsui K, Yoshimoto T, Tsutsui H, Hyodo Y, Hayashi N, Hiroishi K, Kawada N, Okamura H, Nakanishi K, and Higashino K (1997). Propionibacterium acnes treatment diminishes CD4 + NK1.1 + T cells but induces type I T cells in the liver by induction of IL-12 and IL-18 production from Kupffer cells. J Immunol 159:97-106.

Mitchell R, Bacher M, Bernhagen J, Pushkarskaya T, Seldin $M F$, and Bucala R (1995). Cloning and characterization of the gene for mouse macrophage migration inhibitory factor (MIF). J Immunol 154:3863-3870.

Miyake S, Makimura M, Kanegae Y, Harada S, Sato Y, Takamori K, Tokuda C, and Saito I (1996). Efficient generation of recombinant adenoviruses using adenovirus DNAterminal protein complex and a cosmid bearing the full-length virus genome. Proc Natl Acad Sci USA 93:1320-1324.

Mizoguchi Y, Sakagami Y, Kuboi H, Kobayashi K, and Yano I (1988). Effects of the polysaccharide chain of lipopolysaccharide in an experimental massive hepatic cell necrosis model. Biochem Biophys Res Commun 155:1305-1310.

Mizue $\mathrm{Y}$, Nishihira J, Miyazaki T, Fujiwara S, Chida M, Nakamura K, Kikuchi K, and Mukai M (2000). Quantitation of macrophage migration inhibitory factor (MIF) using the onestep sandwich enzyme immunosorbent assay: Elevated serum MIF concentrations in patients with autoimmune diseases and identification of MIF in erythrocytes. Int J Mol Med 5:397-403.
Nishihira J (2000). Macrophage migration inhibitory factor (MIF): Its essential role in the immune system and cell growth. J Interferon Cytokine Res 20:751-762.

Nishihira J, Koyama Y, and Mizue Y (1998). Identification of macrophage migration inhibitory factor (MIF) in human vascular endothelial cells and its induction by lipopolysaccharide. Cytokine 10:199-205.

Okamura H, Tsutsi H, Komatsu T, Yutsudo M, Hakura A, Tanimoto T, Torigoe K, Okura T, Nukada Y, and Hattori K (1996). Cloning of a new cytokine that induces IFN-gamma production by T cells. Nature 378:88-91.

Ostapowicz G and Lee WM (2000). Acute hepatic failure: A Western perspective. J Gastroenterol Hepatol 15:480-488.

Paralkar V and Wistow G (1994). Cloning the human gene for macrophage migration inhibitory factor (MIF). Genomics 19:4851.

Peeters MJ, Patijn GA, Liebert A, Meuse L, and Kay MA (1996). Adenovirus-mediated hepatic gene transfer in mice: Comparison of intravascular and biliary administration. Hum Gene Ther 7:1693-1699.

Sakai M, Nishihira J, Hibiya Y, Koyama Y, and Nishi S (1994). Glutathione binding rat liver $13 \mathrm{~K}$ protein is the homologue of the macrophage migration inhibitory factor. Biochem Mol Biol Int 33:439-446.

Shands JW Jr and Senterfitt VC (1972). Endotoxin-induced hepatic damage in BCG-infected mice. Am J Pathol 67:23-40.

Simpson KJ (1999). Cytokines, for better or worse? Eur J Gastroenterol Hepatol 11:957-966.

Tanaka Y, Takahashi A, Watanabe K, Takayama K, Yahata T, Habu S, and Nishimura T (1996). A pivotal role of IL-12 in Th1-dependent mouse liver injury. Int Immunol 8:569-576.

Tokushige K, Yamaguchi N, Ikeda I, Hashimoto E, Yamaguchi K, and Hayashi N (2000). Significance of soluble TNF receptor-I in acute-type fulminant hepatitis. Am J Gastroenterol 95:2040-2046.

Tsuji H, Harada A, Mukaida N, Nakanuma Y, Bluethmann H, Kaneko S, Yamakawa K, Nakamura SI, Kobayashi KI, and Matsushima K (1997). Tumor necrosis factor receptor p55 is essential for intrahepatic granuloma formation and hepatocellular apoptosis in a murine model of bacterium-induced fulminant hepatitis. Infect Immun 65:1892-1898.

Tsutsui H, Matsui K, Kawada N, Hyodo Y, Hayashi N, Okamura H, Higashino K, and Nakanishi K (1997). IL-18 accounts for both TNF-alpha- and fas ligand-mediated hepatotoxic pathways in endotoxin-induced liver injury in mice. J Immunol 159:3961-1967.

Wada S, Fujimoto S, Mizue Y, and Nishihira J (1997). Macrophage migration inhibitory factor in the human ovary: Presence in the follicular fluids and production by granulosa cells. Biochem Mol Biol Int 41:805-814.

Weiser WY, Temple PA, Witek-Giannotti JS, Remold HG, Clark SC, and David JR (1989). Molecular cloning of a CDNA encoding a human macrophage migration inhibitory factor. Proc Natl Acad Sci USA 86:7522-7526. 